\title{
Mental Health, Resilience and Sports Activity in the Initial Phase of The First COVID-19 Lockdown in Germany
}

\section{Jana Strahler}

University of Giessen: Justus Liebig Universitat Giessen

Konrad Smolinski

KS Sportsworld

Karsten Krüger ( $\square$ karsten.krueger@sport.uni-giessen.de)

University of Giessen: Justus Liebig Universitat Giessen https://orcid.org/0000-0003-1506-8254

Britta Krüger

University of Giessen: Justus Liebig Universitat Giessen

\section{Research Article}

Keywords: COVID-19 pandemic, Mental health, Sports activity, Resilience, Stress

Posted Date: April 21st, 2021

DOI: https://doi.org/10.21203/rs.3.rs-424618/v1

License: (c) (i) This work is licensed under a Creative Commons Attribution 4.0 International License.

Read Full License 


\section{Abstract \\ Background}

Quarantine and social-distancing measures during the COVID-19 pandemic situation resulted in a radical change in lifestyle behaviors. While the reduction of total physical activity is assumed to negatively impact psychological health (higher stress and anxiety levels), regular sports activity during lockdown conditions has beneficial effects on health. Mechanisms are however unclear. The present analysis therefore examined the associations of sports activity with mental health, and assessed whether this is due to a direct effect on experiencing positive emotions and mental health, due to a stress-buffering mechanism, and/or through protecting/enhancing resilience.

\section{Methods}

An online survey, accessible from April 7th to April 30th 2020, gathered data on sports activity (Physical Activity, Exercise, and Sport Questionnaire, BSA), mental health (Patient Health Questionnaire-4, PHQ-4, WHO Wellbeing Index, WHO-5), momentary stress (single item), and resilience (i.e. feeling determined, cheerful, content, and being interested in the things one is doing). The final data set comprised 742 subjects including 534 (72.0\%) women and ranging in age from 16 to 83 years (mean: $28.13 \pm 11.46$ years).

\section{Results}

Across all participants, sports activity was related to higher wellbeing and resilience but lower affective psychopathology. Importantly, all coefficients were below 0.2 indicating only small-sized associations. Moderation analyses confirmed a direct effect of sports activity on affective symptoms and wellbeing. Stress-buffering effects were not confirmed for either outcome but a resilience-protecting effect was seen for both wellbeing and affective psychopathology.

\section{Conclusions}

During the initial phase of the first COVID-19 lockdown, sports activity was associated with better mental health and wellbeing. Besides this direct effect, there was also evidence for a resilience-protecting effect of sport. The assumed stress-buffering effect could not be confirmed. Present findings indicate resilienceprotective mechanisms to be a major contributor to sports beneficial effects on mental health during quarantine. Though, results from this cross-sectional, predominantly female, and convenience sample study must be confirmed in more diverse samples.

\section{Background}


On March $11^{\text {th }}, 2020$, the World Health Organization announced the coronavirus (COVID-19) disease outbreak a pandemic ${ }^{1}$. In response, countries worldwide have had introduced protective measures like (self-)quarantine and physical distancing to decrease cases and prevent the virus' spread. The Federal and State Governments of Germany adopted comprehensive contact restrictions starting on March $10^{\text {th }}$ and a significant tightening of measures around March $27^{\text {th2 }}$. At the beginning of May, the greatest restrictions were gradually lifted. During the summer months, restrictions were rather local and temporary, and of small to moderate degree ${ }^{3,4}$. In the fourth quarter of 2020, measures intensified again. During the first lockdown, attention was soon focused on the psychological impact of the pandemic situation and the confinement measures with most studies reporting on negative psychological effects. Across various populations, anxiety, depression, and stress considerably increased ${ }^{5}$. It is therefore of utmost importance to understand what can be done to mitigate the negative consequences of pandemic situations and quarantine measures.

Lifestyle behaviors like exercise, diet and sleep play major roles in the prevention and treatment of mental illness ${ }^{6}$. The role of regular physical activity and sports activity in mental and physical health is particularly well-researched. A number of meta-analysis show sports activity to aid the prevention and management of mental illness 7,8 . Hence, an active lifestyle and sport are assumed effective means to reduce negative impact of confinement measures ${ }^{9,10}$. According to the model of possible effects of sports activity in the transactional process of disease development, five possible mechanisms were proposed described as the direct, preventive, protective (stress-buffering), resource-creating and resourceprotective effect of sport ${ }^{11}$. One such resource in the context of sport is resilience, i.e. adapting, managing and negotiating adversity. Theory suggests resilient individuals to bounce back from negative experiences quicker and more effectively ${ }^{12}$. While in the past, resilience was primarily understood as a trait, today it is generally accepted that resilience exists on a continuum, resilience may differ across different domains of life and may change over time ${ }^{13}$. The present investigation adopted this theoretical assumption. Regular sports activity, more specifically the related psycho-physiological adaptations, are important contributors and mechanisms of resilience ${ }^{14}$. Resilience, on the other hand, is known to be related to less anxiety/depressive symptoms and more positive emotions ${ }^{15}$. Finally, a body of research confirms the interplay between sports activity, resilience, and mental health ${ }^{16,17}$. Although, the exact directions of action are not yet understood. For the present study, we adopted the above mentioned model of sports' direct and stress-buffering effects on health and additionally considered resilience to be a resource for mental health.

In the context of lifestyle behaviors, current COVID-19-related literature shows that adopted preventive measures resulted in a radical change in lifestyle behaviors ${ }^{18,19}$. For physical and sports activity in particular, physical activities decreased significantly during the lockdown across all age groups $20,2122,23$. This reduction of total physical activity is assumed to have a profoundly negative impact on psychological health (higher stress and anxiety levels) and well-being ${ }^{24,25}$. Data on physical and sport activity during the COVID19 confinement measures from the German general population are however rare 
26-28 and there is only initial evidence for the effects sport may have on reducing distress and fostering mental health during the current pandemic situation. In a large-scale, global cross-sectional study, Brand and colleagues showed that those who exercised almost daily during lockdown conditions had best subjective well-being ${ }^{29}$. Albeit with only small effects, another cross-country study revealed a correlation between change in physical activity from "before" to "during" home confinement and change in wellbeing, life satisfaction and depressive symptoms. Those participants who showed a decrease in their physical activity levels showed poorer psychological health ${ }^{30}$. Another cross-country study provided initial evidence for sport activity (single item measure) to mediate the relationship between depressive symptoms and burden caused by the COVID-19 pandemic ${ }^{31}$. While thus associations between sports behavior and mental health during the COVID-19 pandemic can be assumed, mediating and moderating variables are barely understood. From above summarized research we propose stress and resilience to be such variables.

Hence, the main question for this study was whether sports activity provides an advantage for overcoming the COVID19 pandemic situation and quarantine measures and if so, how. The present analysis therefore examined the associations between sports activity and mental health, and assessed whether this is due to a direct effect on experiencing positive emotions and mental health, due to a stressbuffering mechanism ${ }^{32}$, and/or through protecting/enhancing resilience ${ }^{16}$. Due to differences in stress perception and coping behaviors between men and women ${ }^{33}$, gender-specific associations were assumed and tested.

\section{Methods}

\section{Participants and Design}

Data for this study stems from a larger longitudinal project aiming to characterize the impact of COVID19-related restrictions on physical and sports activity, and subjective well-being. Data herein reports on the initial baseline assessment that started on April $7^{\text {th }} 2020$, about 2 weeks after a variety of stricter lockdown measures were implemented throughout Germany ${ }^{2,4}$. Though the baseline survey was accessible until July $6^{\text {th }}$, only questionnaires completed until April $30^{\text {th }}$ were considered to cover the period with most serious restrictions in mobility. The longitudinal project involved five bi-weekly repeated invitation to take part in a follow-up assessment. This data will be presented elsewhere (Strahler et al., in preparation). This study recruited a convenience sample from the general population and the survey was presented online using the platform SosciSurvey. The study was advertised using university mailing lists and social media. In agreement with the most recent version of the Helsinki declaration, participation was voluntary and informed consent was obtained. Before starting the survey, a study agreement was presented and participants were required to click a checkbox indicating that they agree to the terms. The only further inclusion criteria was being 16 years of age or older. 
Overall, the baseline survey was started 1141 times and completed 901 times (79.0\%). From these 901 data sets, 159 had to be excluded: 37 were questionnaires filled out with "no consent", six questionnaires were filled extremely fast ( $<6 \mathrm{~min}$ and relative speed index $>2$ ), one data set contained responses making a misunderstanding of the questionnaire likely, five data sets provided unreasonable high sports ( $>40 \mathrm{~h} /$ week) or physical activity ( $>98 \mathrm{~h} /$ week, see also below), and 110 records date from the period after May $1^{\text {st }}$. The final data set thus comprised 742 subjects including $534(72.0 \%)$ women and ranging in age from 16 to 83 years (mean: $28.13 \pm 11.46$ years). Two subjects indicated "diverse" as their gender. Both data sets were excluded from gender-specific analyses.

\section{Measures}

The complete survey lasted about 10 to 15 minutes and gathered data on anxious and depressive symptoms, psychological wellbeing, life satisfaction, physical and sports activity, stress, and resilience. This was complemented by sociodemographic and physical health data.

Mental health: Anxious and depressive symptoms were measured by the Patient Health Questionnaire-4 (PHQ-4) ${ }^{34}$. This ultra-brief measure consists of a 2-item depression scale and 2-item anxiety scale with response options ranging from "not at all" $(=0)$ to "nearly every day" $(=3)$. The composite PHQ-4 total score (ranging from 0 to 12) was used in the present report. The total score showed acceptable internal consistency $\left(\mathrm{a}_{\text {Cronbach }}=.78\right)$. Wellbeing was assessed by the WHO Wellbeing Index $(\mathrm{WHO}-5)^{35}$ and the Life Satisfaction Scale (L1) ${ }^{36}$. The 5 items of the WHO-5 (e.g., "In the past two weeks, I have felt cheerful and in good spirits") are to be scored from 5 ("all of the time") to 0 ("none of the time"). A sum score is created and multiplied by 4 to obtain a percentage scale from 0 (absence of well-being) to 100 (maximal well-being). Internal consistency was good herein $\left(\mathrm{a}_{\text {Cronbach }}=.82\right)$. The L1 is a single-item measure recording the cognitive evaluation of one's own quality of life ("All things considered, how satisfied are you with your life these days? ") with an 11-point scale (0 "not at all satisfied" to 10 "completely satisfied"), with moderate re-test stability and adequate construct validity.

Physical and sports activity. Physical and sports activity were examined by means of the Physical Activity, Exercise, and Sport Questionnaire (BSA) ${ }^{37}$ which is based on the Frequency - Intensity - Timeand -Type principle. The BSA records different leisure time and sport activities of the last four weeks. Participants record the frequency and duration (min) of each activity or sport episode. Finally, a "daily activity" index (including job-related and household activities), a "sport activity" index (e.g., swimming, athletic sports, jogging, or yoga), and a "total activity" index (min/week) are determined. According to the model proposed above and according to research showing moderate- and vigorous-intensity activity to be more beneficial for mental health as compared to low intensity activity ${ }^{38}$, present analyses focused on the sports activity index capturing planned, structured, and repetitive activities performed to improve or maintain physical fitness. Daily and total activity were reported for descriptive purposes but did not enter multivariate analyses. 
Stress and resilience: To examine general current stress levels (not specific to the current lockdown situation) participants rated a single item "I fell stressed out" on a visual analogue scale with the poles 0 (not at all) and 100 (completely). Single-item measures of stress are often used when stress has to be monitored repeatedly and in large-scale survey research. Their content, criterion, and construct validity were shown to be satisfactory ${ }^{39}$. Resilience was assessed via four recently proposed items to capture momentary feelings of resilience, i.e. felling determined, cheerful, content, and being interested in the things one is doing ${ }^{40}$. While the authors proposed 7-point Likert scales, we decided to adapt responding to the stress item and also used visual analogue scales with the poles 0 (not at all) and 100 (completely). The mean score was created for analyses and this measure showed good reliability $\left(\mathrm{a}_{\text {cronbach }}=.84\right.$ ).

Confounders: Besides gender (male, female, diverse), age (yrs), relationship status (single, current partnership), number of children $(0,1,2,3$, more than 3$)$, educational level (no or primary or still in school, lower secondary, higher secondary, higher), and subjective socioeconomic status (MacArthur ladder ${ }^{41}$ ) were recorded to describe the sample.

\section{Statistical analysis}

First, descriptive statistics were performed for men and women separately. In a next step, we tested all observed variables regarding their distribution features. As not all of the variables were normally distributed, Mann-Whitney-U-Test was used to identify differences between men and women regarding mental health scores (WHO-5, PHQ-4, L-1), physical activity (total physical activity, activity of daily living, sports activity) resilience and stress experience.

We further used Spearman's rank correlation coefficients to determine the relationship between mental health (as indicated by WHO-5, L-1, and PHQ-4), physical activity (activity of daily living, sports activity, total activity), resilience and feelings of stress for women and men separately.

Lastly, we analyzed the impact of resilience, sports activity and feelings of stress as potential predictors of mental health and wellbeing using multiple regression analyses for the whole group as well as for men and women separately. We further added four product terms to the model in order to test for interactions between sports activity, resilience and stress. Prior to the analysis, we $z$-standardized all variables of interest. We further checked for multicollinearity. VIF was always $<10$. All residuals were normally distributed as indicated by normal P-P-plots. There was no clear distribution pattern in scatterplot of residuals versus predicted values indicating homoscedasticity. Durbin Watson test revealed no autocorrelation between the included regressors.

JASP, version 14.0, was used for statistical analysis and partly for graphical illustration. $P$ values $<.05$ will be considered significant. The Benjamini-Hochberg procedure (with $\delta=.05$ ) was used in order to decrease false discovery rate (FDR) in multiple hypothesis testing.

\section{Results}


Table 1 provide the characteristics of the study population. For women, the level of mental health was represented through the WHO-5 scoring $57.45 \pm 18.69$, the PHQ-4 being $2.96 \pm 2.37$, and the L- 1 being $6.77 \pm 1.85$ on average. For men, the present data revealed a WHO-5 scoring $60.04 \pm 18.86$, the PHQ-4 being $2.26 \pm 2.10$, and the L-1 being $6.93 \pm 1.76$ on average. A significant lower PHQ-4 was revealed for men compared to women $(p<.001$, Tab. 1$)$.

\section{Descriptives: Physical activity, resilience and stress experience}

The average total activity of women was $957.23 \pm 770.78$ [min/week], whereby $631.89 \pm 698.1$ [min/week] were accounted for by the activity of daily living and $325.39 \pm 268.24$ [min/week] for sports activities. Men showed a total activity of $993.24 \pm 851.84$ [min/week], whereby $636.66 \pm 770.59$ [min/week] were accounted for by the activity of daily living and $356.59 \pm 293.03$ [min/week] for sports activities. The present data revealed no significant differences regarding the different modalities of physical activity between men and women (all $p>.05$, Tab. 1)

Table 1. Descriptive data on the study population $(n=742)$ 


\begin{tabular}{|c|c|c|c|}
\hline Variable & Total sample $(\mathrm{N}=742)$ & $\begin{array}{l}\text { Women } \\
(n=534)\end{array}$ & $\begin{array}{l}\text { Men } \\
(n=206)\end{array}$ \\
\hline \multirow[t]{2}{*}{ Age, yrs } & $28.13 \pm 11.46$ & $26.71 \pm 9.97$ & $31.86 \pm 14.02$ *** \\
\hline & $(16-83)$ & $(16-76)$ & $(16-83)$ \\
\hline \multicolumn{4}{|l|}{ Relationship status, n (\%) } \\
\hline Single & $379(51.1)$ & $275(51.5)$ & $103(50.0)$ \\
\hline solid relationship & $363(48.9)$ & $259(48.5)$ & $103(50.0)$ \\
\hline \multicolumn{4}{|l|}{ Children, n (\%) } \\
\hline No & $598(80.6)$ & $441(82.6)$ & $155(75.2)$ * \\
\hline 1 & 85 (11.5) & $59(11.0)$ & $26(12.6)$ \\
\hline 2 & $48(6.5)$ & $25(4.7)$ & $23(11.2)$ \\
\hline 3 & $10(1.3)$ & $8(1.5)$ & $2(1.0)$ \\
\hline$>3$ & $1(0.1)$ & $1(0.2)$ & $0(0.0)$ \\
\hline \multicolumn{4}{|l|}{ Education, n (\%) } \\
\hline none, still in school, primary & $29(3.9)$ & $21(3.9)$ & $8(3.9)$ ** \\
\hline lower secondary & $50(6.7)$ & $28(5.2)$ & $22(10.7)$ \\
\hline higher secondary & $373(50.3)$ & $288(53.9)$ & $83(40.3)$ \\
\hline Higher & $290(39.1)$ & 197 (36.9) & $93(45.1)$ \\
\hline \multirow[t]{2}{*}{ Subjective social status (1...10) } & $5.94 \pm 1.41$ & $5.88 \pm 1.38$ & $6.09 \pm 1.46$ \\
\hline & $(2-9)$ & $(2-9)$ & $(2-9)$ \\
\hline \multicolumn{4}{|l|}{ Mental health } \\
\hline \multirow[t]{2}{*}{ PHQ-4 (0...12) } & $2.77 \pm 2.32$ & $2.96 \pm 2.37$ & $2.26 \pm 2.10 * \star \star$ \\
\hline & $(0-12)$ & $(0-12)$ & $(0-12)$ \\
\hline \multirow[t]{2}{*}{ WHO-5 (0...100) } & $58.14 \pm 18.76$ & $57.45 \pm 18.69$ & $60.04 \pm 18.86$ \\
\hline & $(0-100)$ & $(0-100)$ & $(0-100)$ \\
\hline \multirow[t]{2}{*}{ L1 (0...10) } & $6.81 \pm 1.83$ & $6.77 \pm 1.85$ & $6.93 \pm 1.76$ \\
\hline & $(1-10)$ & $(1-10)$ & $(1-10)$ \\
\hline \multicolumn{4}{|l|}{ Predictors and moderators } \\
\hline Stress $(0 . . .100)$ & $38.26 \pm 28.64$ & $38.93 \pm 28.66$ & $36.65 \pm 28.59$ \\
\hline
\end{tabular}




\begin{tabular}{|clll|} 
& $(0-100)$ & $(0-100)$ & $(0-100)$ \\
\hline Resilience $(0 \ldots .100)$ & $67.34 \pm 19.54$ & $65.94 \pm 19.83$ & $71.04 \pm 18.29 * *$ \\
& $(0-100)$ & $(2-100)$ & $(0-100)$ \\
\hline BSA, min/week & & & \\
\hline activity of daily living & $632.37 \pm 717.73$ & $631.89 \pm 698.10$ & $636.66 \pm 770.59$ \\
& $(0-5725)$ & $(0-5725)$ & $(1-5585)$ \\
\hline sports activity & $333.22 \pm 275.60$ & $325.34 \pm 268.24$ & $356.59 \pm 293.03$ \\
& $(0-1530)$ & $(0-1485)$ & $(0-1530)$ \\
\hline total activity & $965.58 \pm 793.38$ & $957.23 \pm 770.79$ & $993.24 \pm 851.84$ \\
& $(15-5835)$ & $(20-5835)$ & $(15-5765)$ \\
\hline${ }^{*} \mathrm{p}<0.05,{ }^{* *} \mathrm{p}<0.01,{ }^{* * *} \mathrm{p}<0.001$ & & & \\
\hline
\end{tabular}

\section{Bivariate associations between physical activity scores, mental health (reflected by L-1, WHO-5, PHQ-4), resilience and stress experience in men and women}

The three activity indices were correlated with the mental health scores, resilience and stress experience in the entire group, as well as in men and women separately (for a detailed depiction, see Fig. 1). In the total sample, the subcategory total activity significantly correlated with WHO-5 $(r=.134, p<.001)$ and PHQ-4 $(r=-.125, p<.001)$. Sports activity though showed a significant correlation with resilience $(r=.131$, $p<.001)$, WHO-5 $(r=.174, p<.001)$, and PHQ-4 $(r=-.163, p<.001)$. There were no correlations with activity of daily living (all $p>.05$ ).

For women, we found significant correlations between sports activity with WHO-5 ( $r=.205, p<.001)$, PHQ-4 $(r=-.182, p<.001)$, and resilience $(r=.156, p<.001)$. The activity of daily living score significantly correlated with PHQ-4 only $(r=-.149, p<.001)$. For men, we found no significant associations between the activity parameters, mental health, wellbeing, resilience and stress. All correlations for the whole group and by gender can be found in Fig. 1A.

\section{Multivariate analysis: Prediction of the mental health by resilience, stress experience and sports activity during lockdown}

Multiple regressions analyzed whether mental health (represented by L1, WHO-5, PHQ-4) was predicted by resilience, stress experience, sports activity, as well as their interactions. Regarding life satisfaction, increases in L1-scores correlated significantly with increases in resilience $(\beta=.648, p<.001)$, as well as decreases in stress experience $(\beta=-.109, p<.001)$ The inclusion of the product terms did not explain significant additional variance in the L1 score $\left(R^{2}\right.$ Change $=.003, F$ Change $\left.(4,734)=1.032, p>.05\right)$, 
revealing no significant interactions. The total variance explained by the model as a whole was $R^{2}=.460$, $F(3,738)=211.291, p<.001$. Results further showed that the WHO-5 score was significantly moderated by resilience $(\beta=.677, p<.001)$, stress $(\beta=-.212, p<.001)$ and sports activity $(\beta=.060, p=.014)$. The inclusion of product terms further revealed an interaction between resilience and sports activity $(\beta=-.064$, $p=.009$ ) reflecting that wellbeing of those participants with a low resilience and low sports activity is impaired the most (see Fig. 2A). However, this model explained only slightly more variance in the WHO-5 score $\left(R^{2}\right.$ Change $=.006, F$ Change $\left.(4,734)=2.436, p=.046\right)$. The total variance explained by the model as a whole was $R^{2}=.569, F(3,738)=326.882, p<.001$. Moreover, increases in PHQ-4 scores correlated significantly with decreases in resilience $(\beta=-.593, p<.001)$, decreases in sports activity $(\beta=-.063, p=$ $.017)$ and increases in stress $(\beta=.263, p<.001)$. The inclusion of the product terms did explain only little additional variance in the PHQ-4 score $\left(R^{2}\right.$ Change $=.014, F$ Change $\left.(4,734)=5.201, p<.001\right)$ revealing significant interactions between stress experience and resilience $(\beta=-.093, p<.001)$ reflecting a lower resilience as well as a higher stress experience during lockdown being associated with more anxious and depressive symptoms. Furthermore, we found a significant interaction between sports activity and resilience ( $\beta=.073, p=.006$ ) (Fig. $2 \mathrm{~B}$ ). Thus, low sports activity and low resilience was associated with highest symptoms of depression and anxiety. The total variance explained by the model as a whole was $R^{2}=.490, F(3,436)=237.97, p<.001$.

For women, life satisfaction was significantly predicted by stress experience $(\beta=-.146, p<.001)$ and resilience $(\beta=.655, p<.001)$. The inclusion of product terms revealed no significant interaction $\left(R^{2}\right.$ Change $=.004, F$ Change $(4,526)=.967, p=.425)$. The total variance explained by the full model was $R^{2}$ $=.486, F(3,530)=167.28, p<.001$. For psychological wellbeing, we found significant associations for stress experience $(\beta=-.234, p<.001)$ and resilience $(\beta=.665, p<.001)$ but not sports activity $(\beta=.055, p$ $=.055)$. The inclusion of product terms indicated a slight interaction between sports activity and resilience, but this was not significant after FDR correction ( $\beta=.059, p=.047), R^{2}$ Change $=.005, F$ Change $(4,526)=1.592, p=.175$. The total variance explained by the model as a whole was $R^{2}=.570$, $F(3,530)=236.23, p<.001$. With regard to affective symptoms, we found significant associations for stress experience $(\beta=.278, p<.001)$ and resilience $(\beta=-.586, p<.001)$ in women. Again, sports activity did not appeared as a significant predictor in the female sample $(\beta=-.059, p=.059)$. The inclusion of product terms revealed a significant interaction between resilience and stress experience $(\beta=-.110, p<$ $.001), R^{2}$ Change $=.015, F(4,526)=3.927, p=.004$. The interaction between sports activity and resilience failed to reach significance. The total variance explained by the model as a whole was $R^{2}=.497, F(3$, $530)=176.282, p<.001$. Overall, analyses showed that especially resilience and stress experience are the most relevant predictors of mental health in women. Furthermore, sports activity had a small wellbeingenhancing effect in women with low resilience.

For men, life satisfaction was significantly predicted by resilience $(\beta=.647, p<.001)$ but not stress ( $\beta=$ $.006, p=.907)$. The inclusion of product terms revealed no significant interaction, $R^{2}$ Change $=.015, F$ Change $(4,200)=1.336, p=.258$. The total variance explained by the full model as a whole was $R^{2}=$ 
$.409, F(3,204)=48.752, p<.001$. For psychological wellbeing, we found significant associations for stress experience $(\beta=-.162, p<.001)$ and resilience $(\beta=.712, p<.001)$ but not sports activity $(\beta=.080, p$ $=.081)$. The inclusion of product terms suggested an interaction between sports activity and resilience, but this did not remain significant after correction $(\beta=-.101, p=.032), R^{2}$ Change $=.012$, F Change $(4,200)$ $=1.451, p=.291$. The total variance explained by the model as a whole was $R^{2}=.569, F(3,204)=91.908$, $p<.001$. With regard to affective symptoms, we found significant associations for stress experience $(\beta=$ $0.229, p<.001)$ and resilience $(\beta=-0.592, p<.001)$. The interaction between sports activity and resilience was not significant after adjusting for multiple testing $(\beta=.115, p=.032), R^{2}$ Change $=.441, F$ Change( 4 , $200)=1.470, p=.231$. The total variance explained by the model as a whole was $R^{2}=.441, F(3,204)=$ $55.330, p<.001$. The present data revealed, that in men with lower resilience, sports activity was associated with fewer symptoms of depression and anxiety and better psychological wellbeing.

\section{Discussion}

\section{Summary of main findings}

The main question for this study was whether sports activity provides an advantage for overcoming a pandemic situation and quarantine measures. Therefore, sports activities' direct effects on mental health, stress-buffering, and resilience-protecting effects were examined in a cross-sectional study during the first COVID19-related lockdown in April/May 2020. In addition, gender differences were tested. In this convenience sample, women showed higher anxiety and depressive symptoms but similar stress, resilience and activity scores. Across all participants, sports activity was related to higher wellbeing and resilience but lower affective psychopathology. Moderation analyses confirmed this direct effect of sports activity on affective symptoms and psychological wellbeing, but not life satisfaction. Furthermore, we found a moderation between sports activity and resilience. In those with lower resilience, higher sports activity was linked to better wellbeing and reduced affective psychopathology. Stress-buffering effects were not confirmed for either outcome and a resilience-protecting effect was seen for psychological wellbeing and affective symptoms, but not life satisfaction. Importantly, mental health levels of those participants with higher resilience were never reached. The gender-separated analyses showed that, in women, lower resilience accompanied by higher stress experience was associated with highest depressive symptoms and symptoms of anxiety. The resilience-protecting effect of sports activity was no longer evident in the gender-separated analyses.

\section{Direct effects of sports activity on mental health, stress and resilience during lockdown}

The direct effect of sports activity on mental health, feelings of stress and resilience is well documented in the general literature $7,8,15$. In contrast to previous reports, however, effects can only be regarded smallsized. This might be traced back to the heterogeneity of sports activity levels in the current sample without specifying intensity of such activities. Here, moderate to vigorous intensity seems more beneficial for mental health than light intense activities ${ }^{42}$. However, studies conducted during COVID19-related mitigation measures confirm these rather low correlations $24,25,31$. Unexpectedly, there was no correlation 
between sports activity level and stress in the present sample. This contrast previous reports of the stress-reducing effect of physical activity and exercise ${ }^{43}$. Relatively low stress levels (about 38 on a scale from 0 to 100) in our sample could have resulted in a floor effect. Studies in individuals at risk for heightened stress during a pandemic situation are still pending and need to confirm this assumption of critical stress levels beyond which sports activity shows its beneficial effects. In chronically stressed populations, such as caregivers, stress is linked to generally low physical activity levels ${ }^{44}$. While this may be understood as activity's "open potential", that is highly stressed individuals might benefit more from high activity, such populations are also less likely to initiate physical and sports activity ${ }^{45}$. Understanding possible barriers for mental health-enhancing activity levels remains an open task for future research.

Overall, present results confirm sports activity as an effective mean to increase mental health and wellbeing. To contribute to our understanding of moderating variables, the present study considered besides stress - links between sport and resilience.

\section{Prediction of mental health by resilience and stress experience moderated by sports activity}

From the model of possible effects of sports activity in the transactional process of disease development, we also tested sports' stress-buffering capabilities on health and additionally considered resilienceprotective mechanisms. In contrast to our assumptions, there was no indication of a stress-buffering mechanism, neither for measures of wellbeing nor affective psychopathology. This also contradicts larger epidemiological studies ${ }^{46}$ as well as recent COVID19-related research where overall physical activity level was predictive of general stress with a medium effect size ${ }^{32}$. What present data, however, showed was a resilience-protecting mechanism for sports activity on psychological wellbeing and affective symptoms. In participants with higher resilience scores, mental health was unrelated to sport activity. High-resilient individuals always scored above the group mean. In those with lower resilience scores, sports activity appears to have a resilience-protecting effect in addition to the direct effect on mental health. Different psychobiological mechanisms are proposed to underlie such an effect. There is evidence for physical fitness to optimize the neuroendocrine and physiological stress response, to promote an anti-inflammatory state, and to improve certain neuronal processes (e.g., neuronal plasticity or the expression of growth factors) ${ }^{47-51}$. In addition, regular sports activity results in gains in selfregulation ${ }^{52}$ and provides another coping resource via social support, self-efficacy or better sleep quality 53 . This line of research highlights the potential of sports activity for mental health, especially during times of crisis.

As also noted above, when considering the positive effects of physical and sports activity on mental health, it is also important to keep in mind that the initiation or maintenance of activity depends on the individual's mental state and condition ${ }^{45}$. Psychological stress levels (and thus lower mental well-being) predicted lower physical and sports activity as well as higher sedentary time. Besides evidence for this behavioral inhibition effect under stress, there is also evidence for stress-related behavioral activation. Some studies suggest increased activity levels under stress. Here, it can be concluded that activity was 
used as a coping strategy to better manage the stress load. Studies that examined activity levels before as compared to during the pandemic did so only retrospectively $20,2122,2329,30$. This literature speaks more in favor of the behavioral inhibition effect under pandemic stress. Such designs do, however, not allow for causal inferences. Up to date, only a handful of studies with more than one measurement time point have been published. A study in Chinese children and adolescents examined changes in moderateto-vigorous physical activity levels before and during the COVID-19 pandemic and showed a $435 \mathrm{~min}$ reduction on average ${ }^{54}$. Another study in 70 Chinese adults made use of their previous cross-sectional study conducted in 2019 and showed a decline in all types of physical activity ${ }^{55}$. A third study examined 66 Chinese college students who completed a longitudinal survey at least twice ${ }^{56}$. This study showed that maintaining physical activity directly alleviated e general negative emotions. Maximal health buffering effects were seen at weekly physical activity levels equivalent to about 80 min of moderate or 45 min of vigorous activity. Thus, keeping an active lifestyle and maintaining sports activity maybe coping strategies associated with reduced distress and better mental health.

The present analyses additionally showed a differential effect of sports activity on life satisfaction compared to psychological well-being measured with the WHO-5. Though closely linked, life satisfaction and well-being can be considered as independent dimensions that make up mental health ${ }^{57}$. In the present case, life satisfaction assessment was based on the individual's own cognitive judgments of the factors that he/she considered to be most valuable. Well-being assessment was based on rather specific variables like feeling cheerful, relaxed or fresh. The WHO- 5 thus measures the affective and hedonistic dimensions of well-being ${ }^{58}$. Overall, mental health is a multi-dimensional construct, with all dimensions sharing unique variance with sports activity.

\section{Gender-specific associations}

Due to gender-related differences in perception and coping behaviors ${ }^{33}$, this study also examined genderspecific associations. Gender-separate analyses no longer hold the main effect of sports activity on wellbeing and affective symptoms. Again, our rather healthy sample and the limited range in mental health impairment may explain the missing effect. Furthermore, the moderation between stress and resilience on depression and anxiety levels was only found among women. Due to the unequal sample size and the relative small sample of men, a sample-size bias must be taken into account in the interpretation. However, the fact that stress had the most impairing effect in women with low resilience corroborates recent research showing meaning and purpose in life to be beneficial for various dimensions of mental health across cultures and life span ${ }^{59}$. Neither among men nor among women, a resilience-enhancing effect of sport could be shown. This contrasts to earlier COVID19-related literature that suggests physical activity to be a more important mental health factor in both genders with slightly higher associations among women ${ }^{24}$. Important to mention is that in the present study all associations were only smallsized.

\section{Limitations}


Some limitations of our study must be considered. First, with our survey procedure we had to rely on selfreported physical and sports activity levels. Several aspects of bias may have accompanied this selfreport, that is social desirability, selective recall or introspective ability. While this study anonymously collected data, thereby reducing social desirability bias, current stress and mental health levels may have limited the individual's ability to assess their behaviors and health status accurately. Physical activity assessment also did not allow to explore different levels of intensity or mode of activity. From previous research, moderate to vigorous intensity is expected to be more beneficial for mental health than light intensities ${ }^{42}$. Another limitation was the cross-sectional design of our study. Having not assessed preCOVID19 mental health and activity levels precluded us to explore the effects of pre-pandemic levels or whether behavioral changes in response to restrictions rather than the current behaviors are more decisive. This could be interesting because Lesser and Niehuis ${ }^{60}$ showed that the positive associations between sports and wellbeing were primarily found in people who had previously been inactive and started sporting activities with the beginning of the COVID19 restrictions. Furthermore, our recruitment procedure could have resulted in some selection bias. Relatively high activity and low stress levels in our sample may underlie small effect sizes and limit generalizability.

\section{Conclusion}

Taken together, this study provides evidence that sports activity was related to higher wellbeing and resilience but lower affective psychopathology during the first COVID19-related lockdown in April/May 2020. This was clearly demonstrated by a moderation effect between sports activity and resilience. People with low resilience in particular benefit from sport activities in terms of their well-being. At the same time it became clear that in women, lower resilience accompanied by higher stress experience was associated with highest depressive symptoms and symptoms of anxiety, and the resilience-protecting effect of sports activity was no longer evident in the gender-separated analyses. Future studies should be longitudinal and include previous activity levels in order to investigate the relationship between sport and well-being in more detail. The gender-specific results should also be examined with the inclusion of other psychological and biological characteristics.

\section{Declarations}

\section{Credit authorship contribution statement}

Jana Strahler: Conceptualization, Data curation, Investigation, Methodology, Project administration, Writing - original draft, Writing - review and editing. Konrad Smolinski: Conceptualization, Data curation, Formal analysis, Investigation. Karsten Krüger: Investigation, Methodology, Project administration, Writing - review and editing. Britta Krüger: Data curation, Investigation, Methodology, Project administration, Writing - original draft, Writing - review and editing.

\section{Declaration of competing interest}


Jana Strahler, Konrad Smolinski, Karsten Krüger and Britta Krüger declare that they have no conflict of interest. There are no disclosures.

\section{Acknowledgments}

We would like to thank all participants who took part in our study.

\section{Funding}

This research did not receive any specific grant from funding agencies in the public, commercial, or notfor-profit sectors.

\section{Availability of data}

All data are available upon request.

\section{References}

1. World Health Organization, W. Timeline of WHO's response to COVID-19.. 2020 [cited 2020 September 28th]; Available from: https://www.who.int/news-room/detail/29-06-2020-covidtimeline.

2. Bundestag D. Gesetz zum Schutz der Bevölkerung bei einer epidemischen Lage von nationaler Tragweite. Bundesgesetzblatt. 2020;1(14):587-92.

3. Bundesregierung. 2020 [cited 2020 September 28th]; Available from: https://www.bundesregierung.de/breg-de/themen/coronavirus.

4. Steinmetz H, Batzdorfer V, Bosnjak M. The ZPID lockdown measures dataset. June, 2020, ZPID Science Information Online.

5. Brooks SK, et al., The psychological impact of quarantine and how to reduce it: rapid review of the evidence. The Lancet 2020.

6. Firth J, et al. A meta-review of "lifestyle psychiatry": the role of exercise, smoking, diet and sleep in the prevention and treatment of mental disorders. World Psychiatry. 2020;19(3):360-80.

7. Schuch FB, Stubbs B. The Role of Exercise in Preventing and Treating Depression. Curr Sports Med Rep. 2019;18(8):299-304.

8. Stubbs B, et al. EPA guidance on physical activity as a treatment for severe mental illness: a metareview of the evidence and Position Statement from the European Psychiatric Association (EPA), supported by the International Organization of Physical Therapists in Mental Health (IOPTMH). European Psychiatry. 2018;54:124-44.

9. Liao Y, Shonkoff ET, Dunton GF, The acute relationships between affect, physical feeling states, and physical activity in daily life: a review of current evidence. Frontiers in Psychology 2015; 6:1975.

10. Arora T, Grey I. Health behaviour changes during COVID-19 and the potential consequences: A minireview. J Health Psychol. 2020;25(9):1155-63. 
11. Fuchs R, Hahn A, Schwarzer R. Effekte sportlicher Aktivität auf Selbstwirksamkeitserwartung und Gesundheit in einer streßreichen Lebenssituation. Sportwissenschaft. 1994;24:67-81.

12. Tugade MM, Fredrickson BL. Resilient individuals use positive emotions to bounce back from negative emotional experiences. J Pers Soc Psychol. 2004;86(2):320-33.

13. Southwick SM, et al., Resilience definitions, theory, and challenges: interdisciplinary perspectives. European journal of psychotraumatology 2014; 5:10.3402/ejpt.v5.25338.

14. Silverman MN, Deuster PA. Biological mechanisms underlying the role of physical fitness in health and resilience. Interface focus. 2014;4(5):20140040.

15. Cohn MA, et al. Happiness unpacked: positive emotions increase life satisfaction by building resilience. Emotion. 2009;9(3):361.

16. Yoshikawa E, Nishi D, Matsuoka YJ. Association between regular physical exercise and depressive symptoms mediated through social support and resilience in Japanese company workers: a crosssectional study. BMC Public Health. 2016;16:553.

17. Wermelinger Ávila MP, et al. The Role of Physical Activity in the Association Between Resilience and Mental Health in Older Adults. J Aging Phys Act. 2018;26(2):248-53.

18. Knell G, et al., Health Behavior Changes During COVID-19 Pandemic and Subsequent "Stay-at-Home" Orders. Int J Environ Res Public Health 2020; 17(17).

19. Cancello R, et al., Determinants of the Lifestyle Changes during COVID-19 Pandemic in the Residents of Northern Italy. Int J Environ Res Public Health 2020; 17(17).

20. Gallè F, et al., Sedentary Behaviors and Physical Activity of Italian Undergraduate Students during Lockdown at the Time of CoViD-19 Pandemic. Int J Environ Res Public Health 2020; 17(17).

21. Pišot S, et al., Maintaining everyday life praxis in the time of COVID-19 pandemic measures (ELPCOVID-19 survey). Eur J Public Health 2020.

22. Ruíz-Roso MB, et al., Changes of Physical Activity and Ultra-Processed Food Consumption in Adolescents from Different Countries during Covid-19 Pandemic: An Observational Study. Nutrients 2020; 12(8).

23. Moore SA, et al. Impact of the COVID-19 virus outbreak on movement and play behaviours of Canadian children and youth: a national survey. Int J Behav Nutr Phys Act. 2020;17(1):85.

24. Maugeri G, et al. The impact of physical activity on psychological health during Covid-19 pandemic in Italy. Heliyon. 2020;6(6):e04315.

25. Duncan GE, et al. Perceived change in physical activity levels and mental health during COVID-19: Findings among adult twin pairs. PLoS One. 2020;15(8):e0237695.

26. Schmidt SC, et al. Physical activity and screen time of children and adolescents before and during the COVID-19 lockdown in Germany: a natural experiment. Sci Rep. 2020;10(1):1-12.

27. Mutz M, Gerke M. Sport and exercise in times of self-quarantine: How Germans changed their behaviour at the beginning of the Covid-19 pandemic. International Review for the Sociology of Sport 2020:1012690220934335. 
28. Maertl T, et al. Physical Activity during COVID-19 in German Adults: Analyses in the COVID-19 Snapshot Monitoring Study (COSMO). International Journal of Environmental Research Public Health. 2021;18(2):507.

29. Brand R, Timme S, Nosrat S. When pandemic hits: Exercise frequency and subjective well-being during COVID-19 pandemic. Front Psychol. 2020;11:2391.

30. Ammar A, et al. Effects of home confinement on mental health and lifestyle behaviours during the COVID-19 outbreak: Insight from the ECLB-COVID19 multicenter study. Biology of Sport. 2020;38(1):9-21.

31. Brailovskaia J, et al., The association between depression symptoms, psychological burden caused by Covid-19 and physical activity: An investigation in Germany, Italy, Russia, and Spain. Psychiatry Research 2020:113596.

32. Alsalhe TA, et al., Moderation Effect of Physical Activity on the Relationship Between Fear of COVID19 and General Distress: A Pilot Case Study in Arabic Countries. Frontiers in Psychology 2020; 11(2381).

33. Brougham RR, et al. Stress, sex differences, and coping strategies among college students. Current psychology. 2009;28(2):85-97.

34. Löwe B, et al. A 4-item measure of depression and anxiety: validation and standardization of the Patient Health Questionnaire-4 (PHQ-4) in the general population. J Affect Disord. 2010;122(12):86-95.

35. Staehr J, The use of well-being measures in primary health care-the DepCare project. World Health Organization, Regional Office for Europe: Well-Being Measures in Primary Health Care-the DepCare Project. Geneva: World Health Organization 1998.

36. Beierlein C, et al., Eine Single-Item-Skala zur Erfassung der Allgemeinen Lebenszufriedenheit: Die Kurzskala Lebenszufriedenheit-1 (L-1). 2014: GESIS-Working Papers 2014|33.

37. Fuchs R, et al. Messung der Bewegungs-und Sportaktivität mit dem BSA-Fragebogen. Zeitschrift für Gesundheitspsychologie. 2015;23:60-76.

38. Dunn AL, et al. Exercise treatment for depression: efficacy and dose response. Am J Prev Med. 2005;28(1):1-8.

39. Elo A-L, Leppänen A, Jahkola A. Validity of a single-item measure of stress symptoms. Scandinavian journal of work, environment \& health 2003:444-451.

40. Schwerdtfeger AR, Dick K. Episodes of momentary resilience in daily life are associated with HRV reductions to stressful operations in firefighters: an ambulatory assessment approach using bayesian multilevel modeling. The Journal of Positive Psychology. 2019;14(5):593-602.

41. Adler NE, Stewart J. The MacArthur scale of subjective social status. MacArthur Research Network on SES \& Health. 2007 [cited 2019 November 19]; Available from: http://www.macses.ucsf.edu/Research/Psychosocial/subjective.php.

42. Schuch FB, et al. Exercise as a treatment for depression: a meta-analysis adjusting for publication bias. J Psychiatr Res. 2016;77:42-51. 
43. Gerber M, Pühse U. Do exercise and fitness protect against stress-induced health complaints? A review of the literature. Scand J Public Health. 2009;37(8):801-19.

44. Marquez DX, et al. Physical activity and psychosocial and mental health of older caregivers and noncaregivers. Geriatric Nursing. 2012;33(5):358-65.

45. Stults-Kolehmainen MA, Sinha R. The effects of stress on physical activity and exercise. Sports Med. 2014;44(1):81-121.

46. Aldana SG, et al. Relationships between leisure time physical activity and perceived stress. Perceptual Motor skills. 1996;82(1):315-21.

47. Fragala MS, et al. Neuroendocrine-immune interactions and responses to exercise. Sports Med. 2011;41(8):621-39.

48. Jackson EM, Dishman RK. Cardiorespiratory fitness and laboratory stress: a meta-regression analysis. Psychophysiology. 2006;43(1):57-72.

49. Gleeson $M$, et al. The anti-inflammatory effects of exercise: mechanisms and implications for the prevention and treatment of disease. Nat Rev Immunol. 2011;11(9):607-15.

50. Cotman CW, Berchtold NC, Christie LA. Exercise builds brain health: key roles of growth factor cascades and inflammation. Trends Neurosci. 2007;30(9):464-72.

51. Knaepen K, et al. Neuroplasticity - exercise-induced response of peripheral brain-derived neurotrophic factor: a systematic review of experimental studies in human subjects. Sports Med. 2010;40(9):765801.

52. Oaten $\mathrm{M}$, Cheng $\mathrm{K}$. Longitudinal gains in self-regulation from regular physical exercise. $\mathrm{Br} \mathrm{J}$ Health Psychol. 2006;11(4):717-33.

53. Fox KR. The influence of physical activity on mental well-being. Public Health Nutrition. 2007;2(3a):411-8.

54. Xiang M, Zhang Z, Kuwahara K. Impact of COVID-19 pandemic on children and adolescents' lifestyle behavior larger than expected. Progress in cardiovascular diseases 2020:S0033-0620(20)30096-7.

55. Zheng C, et al. COVID-19 Pandemic Brings a Sedentary Lifestyle in Young Adults: A Cross-Sectional and Longitudinal Study. International Journal of Environmental Research Public Health. 2020;17(17):6035.

56. Zhang Y, et al., Mental Health Problems during the COVID-19 Pandemics and the Mitigation Effects of Exercise: A Longitudinal Study of College Students in China. Int J Environ Res Public Health 2020; 17(10).

57. Seligman MEP. Flourish: A Visionary New Understanding of Happiness and Well-Being. New York: Free Press; 2011.

58. Sischka PE, et al. The WHO-5 well-being index - validation based on item response theory and the analysis of measurement invariance across 35 countries. Journal of Affective Disorders Reports. 2020;1:100020. 
59. Jebb AT, et al. Subjective well-being around the world: trends and predictors across the life span. Psychological science. 2020;31(3):293-305.

60. Lesser IA, Nienhuis CP. The impact of COVID-19 on physical activity behavior and well-being of Canadians. Int J Environ Res Public Health. 2020;17(11):3899.

\section{Figures}

A

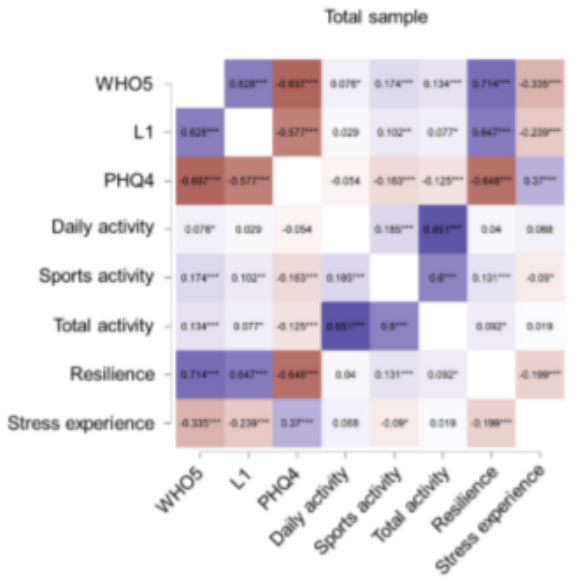

B

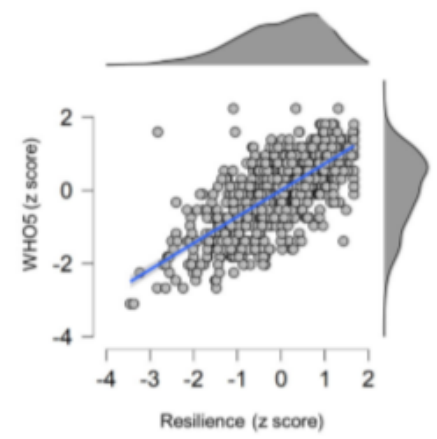

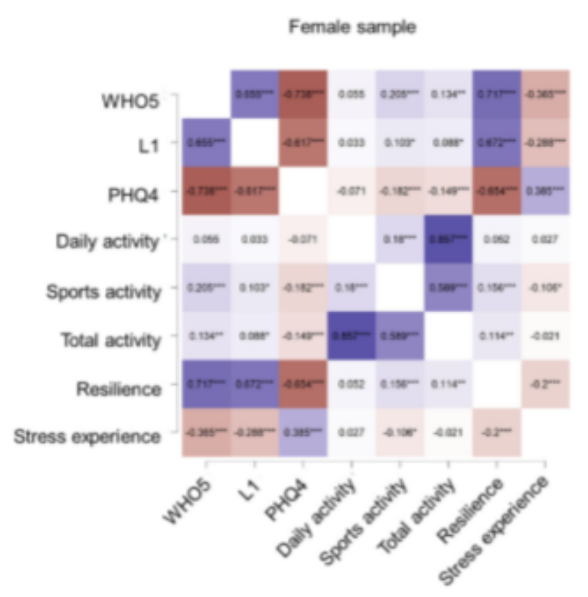

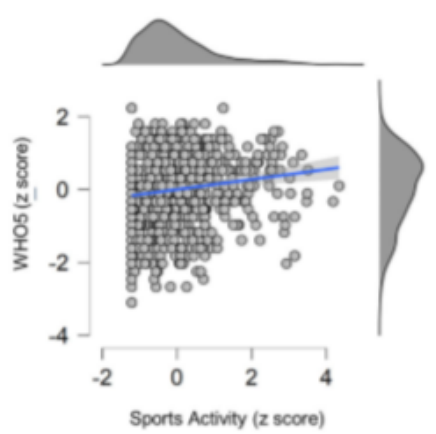

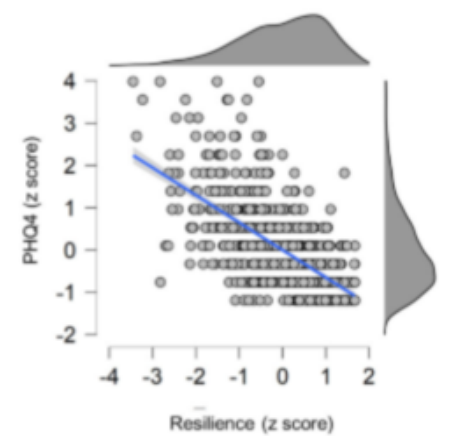

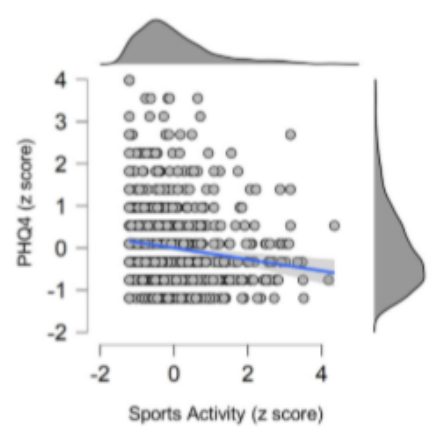

\section{Figure 1}

A Correlation heat map including WHO5, L1, PHQ4, daily activity, sports activity, total activity, resilience and stress experience. Spearman rank correlations are presented. ${ }^{*} p<.05,{ }^{* \star} p<.01,{ }^{* * *} p<.001$. B Associations between WHO5, PHQ4, resilience and sports activity. 
-low sports activity - -high sports activity

A

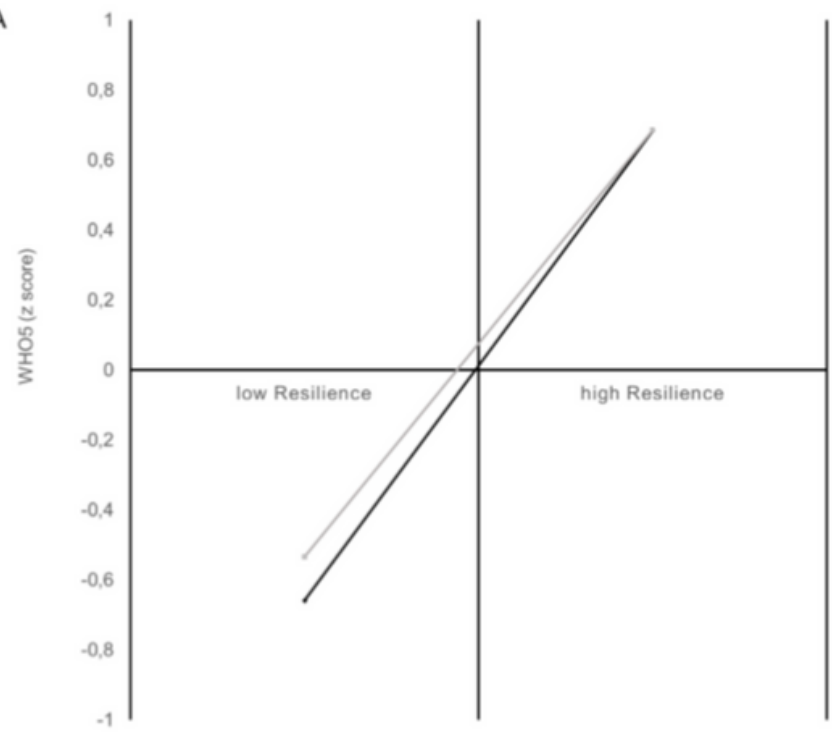

-low sports activity - -high sports activity

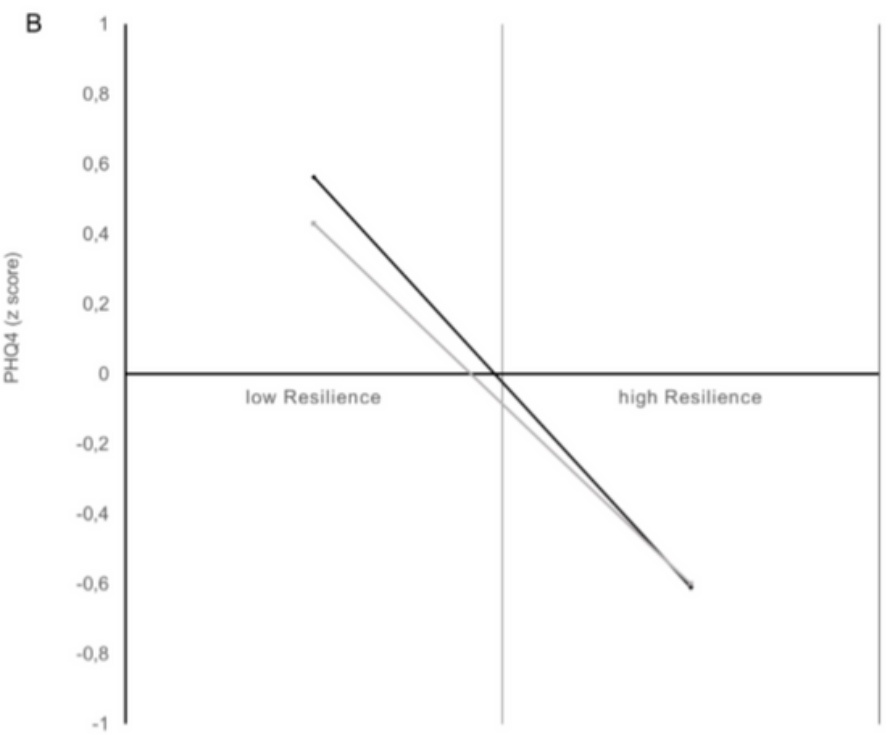

Figure 2

A Associations between low/high sports activity, resilience, and WHO5 B and PHQ4. B Associations between low/high sports activity, resilience, and PHQ4. 